\title{
Nonparametric Change Point Diagnosis Method of Concrete Dam Crack Behavior Abnormality
}

\author{
Zhanchao Li, ${ }^{1,2}$ Chongshi Gu, ${ }^{2,3}$ and Zhongru $\mathrm{Wu}^{3}$ \\ ${ }^{1}$ College of Water Resources and Architectural Engineering, Northwest A\&F University, Yangling 712100, China \\ ${ }^{2}$ State Key Laboratory of Hydrology-Water Resources and Hydraulic Engineering, Hohai University, Nanjing 210098, China \\ ${ }^{3}$ College of Water Conservancy and Hydropower Engineering, Hohai University, Nanjing 210098, China
}

Correspondence should be addressed to Zhanchao Li; qdzxqforever@163.com

Received 2 June 2013; Accepted 14 July 2013

Academic Editor: Xu Zhang

Copyright (C) 2013 Zhanchao Li et al. This is an open access article distributed under the Creative Commons Attribution License, which permits unrestricted use, distribution, and reproduction in any medium, provided the original work is properly cited.

\begin{abstract}
The study on diagnosis method of concrete crack behavior abnormality has always been a hot spot and difficulty in the safety monitoring field of hydraulic structure. Based on the performance of concrete dam crack behavior abnormality in parametric statistical model and nonparametric statistical model, the internal relation between concrete dam crack behavior abnormality and statistical change point theory is deeply analyzed from the model structure instability of parametric statistical model and change of sequence distribution law of nonparametric statistical model. On this basis, through the reduction of change point problem, the establishment of basic nonparametric change point model, and asymptotic analysis on test method of basic change point problem, the nonparametric change point diagnosis method of concrete dam crack behavior abnormality is created in consideration of the situation that in practice concrete dam crack behavior may have more abnormality points. And the nonparametric change point diagnosis method of concrete dam crack behavior abnormality is used in the actual project, demonstrating the effectiveness and scientific reasonableness of the method established. Meanwhile, the nonparametric change point diagnosis method of concrete dam crack behavior abnormality has a complete theoretical basis and strong practicality with a broad application prospect in actual project.
\end{abstract}

\section{Introduction}

China has the most water-conservancy and hydropower projects currently in the world. At the beginning of the 21st century, China entered the peak period of dam construction. There is a group of $320 \mathrm{~m}$-level ultrahigh concrete arc dams completed or under construction in Southwest China. Concrete dam crack and its influence on the structure safety have always been a hot spot and difficulty in the discipline of hydraulic structure. Currently, the study is focused on whether the macrocrack of concrete structure will be expanded under load, which is an important issue for the evaluation of safety performance of concrete structure working with crack [1]. In dam safety monitoring field, as the masses have higher and higher requirements to the safety of water conversancy projects, the problem of concrete dam crack has been more and more highlighted. It has also gradually become a hot spot and difficulty in the study on safety of hydraulic structure to study the transformation of crack behavior in concrete dam due to crack expansion and even its abnormality to cause influence on the safety of concrete structure. Such problem is defined as concrete dam crack behavior abnormality in this paper, and the emphasis is put on the study of diagnosis method of crack behavior abnormality, so as to diagnose the crack abnormality timely and rapidly.

For the hydraulic concrete structure working with crack, Li et al. [2], in combination with mutation theory created the grey cusp mutation model of hydraulic concrete structure crack abnormality. Bao and Yu [3], based on grey differential fitting modeling, created the grey state equation of concrete dam crack, analyzed the relation between the stability of grey state equation and crack subcritical expansion, and proposed the grey system method to distinguish and judge the subcritical expansion of crack. Given that concrete dam crack system is an open, dissipated, and complex nonlinear dynamical 
system, Gu et al. [4] proposed dynamical fuzzy crosscorrelation factor index method to preliminarily explore the dynamic abnormality diagnosis of concrete dam crack behavior. The current study plays an important promotion role in expanding the study thought and promoting discipline crossing, and development and forecasts that applied mathematics theory will have a strong development potential and broad development space in this field.

Deep analysis shows that all abnormality diagnosis methods above are based on the sequence of concrete dam crack mouth opening displacement (CMOD), but, according to numerous researches and investigations, the author finds that whether the CMOD can be taken as the basis for diagnosis of crack behavior abnormality is not deeply studied in the existing literatures, and most of the literatures are based on the fact that CMOD generally is the only monitoring item of concrete dam crack, causing that the existing concrete dam crack abnormality diagnosis method is lack of solid theoretical foundation. Based on this, the author, according to the fracture mechanics fictitious crack model and linear asymptotic superposition assumptions, creates the double crack tip opening displacement criterion of concrete crack behavior abnormality, providing a theoretical basis for the use of CMOD for crack behavior abnormality diagnosis in actual project [5]. Meanwhile, the basis for the existing concrete dam crack behavior abnormality diagnosis method is the parametric statistical model of safety monitoring on concrete dam crack [6], which is established on the basis of this statistical model and in combination with certain or some applied mathematics theories and emphasizes the study from the aspect of information analysis, and it is still required to have a deep analysis on basic issues such as scientificalness and reasonableness of the abnormality diagnosis method.

The parametric statistical model of safety monitoring on concrete dam crack takes hydraulic pressure, temperature, and timeliness as the main influential factors, in which the theoretical basis of crack timeliness component expression is the creep theory of concrete and the component of cyclic load of temperature and so forth is fitted with periodic function, and it expresses the hydraulic component with cubic or quartic polynomial continuous function of water head based on the theory of engineering mechanics [7]. So the parametric statistical model of safety monitoring on concrete dam crack is widely applied in actual project as it can reflect the evolution law of crack behavior truthfully in a certain extent. However, when the abnormality diagnosis of crack behavior is based on the parametric statistical model of safety monitoring on concrete dam crack, as the functional relationship between the influential factors of crack and the crack effect size is set in advance and the stochastic disturbance of model needs to meet the normal consumption, the effect of crack behavior abnormality diagnosis is not reasonable and comprehensive enough. On this basis, closely combining the hot issue in the latest statistics, change point theory, based on the analysis of change point characteristics of concrete dam crack behavior and through the reduction of change point issue, establishment of basic nonparametric change point model, and progressive analysis of basic change point test method as well as the study on multichange point diagnosis method of crack abnormality, the author creates the nonparametric change point diagnosis method of concrete dam crack behavior abnormality in this paper and applies it in the actual project, so as to inspect the scientific reasonableness of nonparametric change point diagnosis method of concrete dam crack behavior abnormality.

\section{Analysis on Change Point Characteristics of Concrete Dam Crack Behavior Abnormality}

Crack behavior abnormality is representation that the crack evolves from stable state to instable state. The abnormity of concrete dam crack behavior is presented as the instability of model structure in crack monitoring model, that is, the dynamic relation between environment size and crack effect size changes suddenly in several time points for some reason or changes continuously and slowly in a certain time interval. For example, according to the existing crack behavior abnormality diagnosis method, the hydraulic component and temperature component in the CMOD are recoverable, while the timeliness component is unrecoverable. Thus, most of the abnormality diagnosis methods of crack behavior are against the timeliness component of CMOD. When the timeliness component meets some conditions, it is thought that the abnormality occurs to the crack behavior, for example, the grey cusp mutation model method [8]. The general form of such methods can be described as first, the general expression of timeliness component of CMOD is defined as

$$
K\left(\theta_{k}\right)=Z_{k}^{T} \alpha, \quad k=1, \ldots, n,
$$

where $\left\{Z_{k}\right\}$ is the influence factor related with timeliness and $\alpha=\left[\alpha_{1}, \alpha_{2}, \ldots, \alpha_{d}\right]^{T}$ is the $d$-dimensional regression parameter of timeliness component; for example, the expression of timeliness component of CMOD is generally $K\left(\theta_{k}\right)=c_{1} \theta_{k}+$ $c_{2} \ln \theta_{k}$.

Then the abnormality of crack behavior in the mode is presented as the regression parameter $\alpha$ changes in some time points that is, in time point $i$, the regression coefficient $\alpha_{1 \sim i}$ determined by samples $1 \sim i$ is significantly different from the regression coefficient $\alpha_{i+1 \sim n}$ determined by samples $i+1 \sim n$ statistically.

The above crack behavior abnormality is diagnosed based on the timeliness component. However, generally the hydraulic pressure and temperature components can also reflect the abnormality of crack behavior. For example, under the same water level and temperature conditions, when the concrete dam crack depth increases, the annual variation amplitude of hydraulic pressure component and temperature component in CMOD will be changed. In the statistical model of CMOD, it is reflected that the regression coefficients of hydraulic pressure component and temperature component are changed; when this change is significant statistically, the crack behavior is abnormal.

For the two given CMOD sample sequences, in order to test whether the crack is abnormal when generating the two sample sequences, the statistical two-sample analysis method can be adopted. In statistics, two-sample analysis method is mainly to analyze whether the probability distribution 
characteristics of two samples are significantly different statistically, for example, the Chow test [9]. For concrete dam crack, assume that, in its monitoring model, the endogenous variable is $y$, exogenous variables are $x_{1}, x_{2}, x_{3}, \ldots, x_{k}$, their $N$ sequence observation values $y_{t}, x_{1 t}, x_{2 t}, x_{3 t}, \ldots, x_{k t}(t=$ $1,2, \ldots, N)$ are from two times with different environments, in which the first $N_{0}\left(N_{0}<N\right)$ observation values are from a certain historical period and the rest $N-N_{0}$ ones are from another period. At this moment, if the data of the pre- $N_{0}$ period, and those of the post- $N-N_{0}$ period are, respectively, used to establish two linear models between $y$ and $x_{1}, x_{2}, x_{3}, \ldots, x_{k}$ :

$$
\begin{array}{r}
Y_{1}=\beta_{0}^{(1)}+\beta_{1}^{(1)} x_{1 t}+\beta_{2}^{(1)} x_{2 t}+\cdots+\beta_{k}^{(1)} x_{k t}+\varepsilon_{t}^{1}, \\
\left(1 \leq t \leq N_{0}\right), \\
Y_{1}=\beta_{0}^{(2)}+\beta_{1}^{(2)} x_{1 t}+\beta_{2}^{(2)} x_{2 t}+\cdots+\beta_{k}^{(2)} x_{k t}+\varepsilon_{t}^{2}, \\
\left(N_{0}+1 \leq t \leq N\right) .
\end{array}
$$

Obviously, if the intercepts of $\beta_{0}^{(1)}$ and $\beta_{0}^{(2)}$ are different or the coefficients of a certain exogenous variable are different, the structural relation between $y$ and $x_{1}, x_{2}, x_{3}, \ldots, x_{k}$ is instable. On the contrary, if the intercepts and coefficients of all exogenous variable are the same, the two formulas are the same model in nature. It is called that there exists a stable structural relation between $y$ and $x_{1}, x_{2}, x_{3}, \ldots, x_{k}$. On this basis, it is only needed to establish one model between all the $N$ observation values between $y$ and $x_{1}, x_{2}, x_{3}, \ldots, x_{k}$ :

$$
Y=\beta_{0}+\beta_{1} x_{1 t}+\beta_{2} x_{2 t}+\cdots+\beta_{k} x_{k t}+\varepsilon_{t}, \quad 1 \leq t \leq N .
$$

It can be seen that the study on structural stability of the model can be converted into the statistical test issue of model parameter in the following form:

$$
\begin{aligned}
& H_{0}: \beta_{i}^{1}=\beta_{i}^{2}, \quad i=0,1, \ldots, k \\
& H_{1}: \exists i, \quad 0 \leq i \leq k \quad \text { s.t. } \beta_{i}^{1} \neq \beta_{i}^{2} .
\end{aligned}
$$

In order to test whether the different hypothesis on the two models is true, start from the original hypothesis that the two regression equations are the same, and then test whether the hypothesis can be refused. If the ordinary least squares method is used to estimate the models (2) and (3), respectively, as there is no limitation condition to the model coefficients, the sum of residual sum of squares of the two equations is used to represent the unconditional residual sum of squares, that is, $\mathrm{ESS}_{\mathrm{UR}}=\mathrm{ESS}_{1}+\mathrm{ESS}_{2}$, in which $\mathrm{ESS}_{1}$ and $\mathrm{ESS}_{2}$, respectively, represent the residual sums of squares of models (2) and (3), they are respectively subject to the $\chi^{2}$ distribution of degrees of freedom of $N_{0}-(k+1)$ and $N-N_{0}-(k+1)$, and ESS ${ }_{\text {UR }}$ is subject to the $\chi^{2}$ distribution of degree of freedom of $N-2(k+1)$. Then the ordinary least squares method is used to evaluate the model (4) and calculate the residual sum of squares $\mathrm{ESS}_{R}$ with condition limit, which is subject to the $\chi^{2}$ distribution of degree of freedom $N-(k+1)$. If we assume that $H_{0}$ is true, then $\mathrm{ESS}_{R}$ will not exceed ESS $\mathrm{UR}_{\mathrm{U}}$ too much. On this basis, the hypothesis $H_{0}$ can be judged whether it is true according to whether the residual sum of squares ESS $=\mathrm{ESS}_{R}-\mathrm{ESS}_{\mathrm{UR}}$ is significant; the test statistics constructed is

$$
F=\frac{\mathrm{ESS} /(k+1)}{\mathrm{ESS}_{\mathrm{UR}} /(N-2(k+1))} .
$$

Obviously, the above test statistics is subject to the $F$ distribution of first degree of freedom $k+1$ and second degree of freedom $N-2(k+1)$. If the structure between $y$ and $x_{1}, x_{2}, x_{3}, \ldots, x_{k}$ is stable, the parameters estimated in the models (2), (3), and (4) should be approximate. Thus the difference between $\mathrm{ESS}_{\mathrm{UR}}$ and $\mathrm{ESS}_{R}$ is tiny; at this moment, $F$ tends to be the smaller value; otherwise, $F$ will be larger. Based on this, the structural stability of the model can be judged accordingly for the significance level $\alpha$ given; if $F<$ $F_{\alpha}(k+1, N-2(k+1)), H_{0}$ is accepted; at this moment, it can be thought that the model structure is stable; that is, no abnormality occurs to the crack behavior at this moment; on the contrary, if $F>F_{\alpha}(k+1, N-2(k+1)), H_{0}$ is refused, and thus the structure between $y$ and $x_{1}, x_{2}, x_{3}, \ldots, x_{k}$ is instable; that is, abnormality occurs to the crack behavior at this moment.

It can be seen that when the abnormality point of concrete dam crack behavior is known, the test analysis method of two-sample issue can be adopted to diagnose whether abnormality occurs to the crack behavior. But in practice, it is difficult to know the moment when abnormality occurs to the crack behavior. When the abnormality point is unknown, the issue on crack behavior abnormality diagnosis is converted into change point issue.

The main study of change point issue is to test and analyze whether the statistical characteristics of a given time sequence are significantly changed at an unknown moment. Statistically, change point $\tau_{0}$ means that some statistical characteristics of the sequence or process change at an unknown moment $\tau_{0}$. Assuming a sample sequence $X_{1}, X_{2}, \ldots, X_{n}$, the corresponding distribution functions are $F_{1}, F_{2}, \ldots, F_{n}$. If there exists $\tau_{0}$, which makes $F_{\tau_{0}+1}$ that differs greatly from $F_{\tau_{0}}$ in some characteristics (such as mean value and position parameter etc.), then $\tau_{0}$ is called a change point of the sequence. More generally, if $X_{1}, X_{2}, \ldots, X_{n}$ are divided into $q$ groups, $\left\{X_{1}, \ldots, X_{\tau_{1}}\right\},\left\{X_{\tau_{1}+1}, \ldots, X_{\tau_{2}}\right\}, \ldots,\left\{X_{\tau_{q}+1}, \ldots, X_{n}\right\}$, with sample distribution in each group relatively stable, while there are mutations at $\tau_{1}, \tau_{2}, \ldots, \tau_{q}$, then $\tau_{1}, \tau_{2}, \ldots, \tau_{q}$ are the $q$ change points of the sequence.

Change point is ubiquitous in nature and society, which reflects the process from quantitative change to qualitative change of things. The issue of change point, whether in theoretical exploration or actual data analysis, is very important, that is, to analyze whether the potential mechanism is changed, when it is changed, and how it is changed based on a group of data; if these problems are ignored, the accuracy of estimation and forecast will be reduced.

For the monitoring sequence of an effect size of concrete dam crack, for example, the CMOD monitoring sequence $X_{1}, X_{2}, \ldots, X_{n}$, whether abnormality occurs to the crack behavior can be expressed as the original hypothesis 
$H_{0}: X_{1} \stackrel{D}{=} X_{2} \stackrel{D}{=} \ldots \stackrel{D}{=} X_{n}$ is true or alternative hypothesis $H_{1}: \exists 1 \leq i, j \leq n$ s.t. $X_{i} \stackrel{D}{\neq} X_{j}$ is true; that is, whether the distribution of CMOD sequence is changed somewhat, while the distribution change forms are diversified in theoretical exploration and practice. The distribution of observation value is changed according to a certain law and is changed to another law at an unknown moment, and this moment is the change point. Or the moment of specific change cannot be found out at all, and only the distribution law of observation is changed progressively just at the beginning. It is also possible that the sample distribution relies on a spatial parameter, while this mutation or progressive change relies on the position or interface in the space. Another possibility is that the distribution law of samples will be changed with change of system condition or an element in the system, and then the mutation or progressive change will rely on a specific nontime and space variable. Generally, the "structural change" in the parametric statistical model is often represented as the change of specific parameter $\theta$. Another "structural change" is not completely decided by the change of some parameters, such issues are of diversified forms and their models are also relatively complex. The change point study on concrete dam crack behavior abnormality in this paper is mainly to detect whether the statistical behavior of the crack is significantly fluctuated, especially to detect whether the crack fluctuation exceeds its safety control range. When the crack is changed qualitatively (mainly exceeding the warning line of crack control), it is hoped that early warning can be released in time to avoid safety accident of the dam.

\section{Construction of Concrete Dam Crack Behavior Change Point Diagnosis Method}

It can be known from the above analysis on characteristics of concrete dam crack behavior abnormality that whether abnormality occurs to the crack behavior can be diagnosed with change point theory. According to the prior information depended, the existing change point theory diagnosis methods can be mainly classified into [10] parametric, semiparametric, and nonparametric diagnosis methods. In the study of change point issues, due to the uniqueness of nonparametric statistics; that is, no background of sample distribution is required, in recent years, the nonparametric methods in study on change point issue have been very active and their contents are also very rich. This paper is focused on the nonparametric change point study of concrete dam CMOD sequence, so as to establish the nonparametric diagnosis method of concrete dam crack behavior abnormality.

3.1. Reduction of Change Point Issue. Any characteristic of the random sequence is determined by its probability distribution, that is, by the probability measure in a functional space. Therefore, the change of any characteristic of the random sequence is the change of its probability distribution generally. Based on this, it can be thought that the random sequence with the change point is "bonded" by the sequence fragments with different probability distributions [10], while the any-probability distribution of random sequence is decided by a limited-dimension distribution set meeting the conditions of consistency [11]. Therefore, any change of the probability characteristics is the product of some change of any limited-dimension distribution. Consider the following single change point model: assume $X=\left\{x_{n}\right\}_{n=1}^{\infty}$ and $Y=\left\{y_{n}\right\}_{n=1}^{\infty}$ are two strictly stationary random sequences, command $1 \equiv \tau_{0}<\tau_{1}<\tau_{2}<\cdots<\tau_{k}, k<\infty$, and define

$$
\begin{aligned}
F_{\tau_{0}, \tau_{1}, \ldots, \tau_{k}}^{\prime}\left(u_{0}, u_{1}, \ldots, u_{k}\right) & \triangleq F^{\prime}(\cdot) \\
& =P\left(x_{\tau_{0}} \leq u_{0}, x_{\tau_{1}} \leq u_{1}, \ldots, x_{\tau_{k}} \leq u_{k}\right), \\
F_{\tau_{0}, \tau_{1}, \ldots, \tau_{k}}^{\prime \prime}\left(u_{0}, u_{1}, \ldots, u_{k}\right) & \triangleq F^{\prime \prime}(\cdot) \\
& =P\left(y_{\tau_{0}} \leq u_{0}, y_{\tau_{1}} \leq u_{1}, \ldots, y_{\tau_{k}} \leq u_{k}\right) .
\end{aligned}
$$

Assume $F^{\prime}(\cdot) \neq F^{\prime \prime}(\cdot)$ and consider the sequence $Z=$ $\left\{z_{n}\right\}_{n=1}^{\infty}$, in which

$$
z_{n}= \begin{cases}x_{n}, & n \leq n_{1}, \\ v_{n}, & n_{1}<n<n_{2}, \\ y_{n}, & n_{2} \leq n,\end{cases}
$$

where $\left\{v_{n}\right\}$ is any sequence. Then it is thought that $Z$ is the sequence with a change point; that is, its $(k+1)$ dimension distribution function has a change point. Here $n_{1}$ and $n_{2}$, respectively, represent the moment when the change point begins and ends, and interval $\left(n_{1}, n_{2}\right)$ is the length of change point transient process. Assume $n_{1}=\left[\theta_{1} N\right], n_{2}=\left[\theta_{2} N\right]+1$ and $0<\theta_{1} \leq \theta_{2}<1$, where $N$ is the sample capacity. If $\theta_{1}=\theta_{2}$, it is thought that the change point is a mutation change point; if $\theta_{1}<\theta_{2}$, it is thought that the change point is a progressive change point. Similarly, corresponding multichange point issues can be defined.

There are varieties of change point issues, and the different probability characteristics of stationary random sequence can be defined as the corresponding change point issues. Thus, only with the help of all finite-dimension distributions can the random sequence be fully described. Therefore, for the purpose of theoretical analysis and practical application of the change point issue, it is necessary to refine a basis change point type from so complex and various change point issues, so that other types of change point issues can be reduced to such basic type conveniently. It is thought that sequence $Z$ with a change point is a basic type of change point if the mathematical expectation of sequence $Z$ changes, that is,

$$
E\left(z_{n}\right)= \begin{cases}a^{\prime}, & n \leq n_{1} \\ a_{n}, & n_{1}<n<n_{2} \\ a^{\prime \prime}, & n_{2} \leq n,\end{cases}
$$

where $a^{\prime} \neq a^{\prime \prime}$ and $\left\{a_{n}\right\}$ is any sequence.

The method to diagnose the change point of the formula (9) is a basic change point diagnosis rule. Generally, in this basic type of change point, other characteristic parameters of the random sequence also may be changed; here other 
parameters are called redundant parameters. Meanwhile, study shows that [10], as long as enough diagnosis sequence $\left\{v_{t}^{j}\right\}$, is introduced; the test of any distribution function parameter change in sequence $Z=\left\{z_{n}\right\}_{n=1}^{\infty}$ can be reduced to a basic type of change point test; that is, the reduction of change point issue makes it possible to adopt the same change point test method to test any information involved in the characteristics of random sequence change point as long as the basic change point test rule is analyzed.

3.2. Basic Nonparametric Change Point Model. Generally, the a posteriori change point problem can be regarded as the estimation problem of parameter vector. Assuming $\theta=$ $\left(\theta_{1}, \theta_{2}, \ldots, \theta_{k}\right), k \geq 1$ is an unknown parameter vector and $0 \equiv \theta_{0}<\alpha \leq \theta_{1} \leq \theta_{2} \leq \cdots \leq \theta_{k} \leq \beta<\theta_{k+1} \equiv 1$ satisfies where $0<\alpha<0.5<\beta$ and $\alpha$ and $\beta$ are two prior constants. Assuming $\phi_{\theta}(t), t \in[0,1]$ is a determined parameter family, two types of random sequence family in the probability space $\left(\Omega, F, P_{\theta}\right)$ should be considered:

$$
\begin{gathered}
\left\{X^{N}\right\}, X^{N}=\left\{x^{N}(n)\right\}_{n=1}^{N}, \quad N>\left[\frac{1}{\alpha}\right] \vee\left[\frac{1}{1-\beta}\right], \\
\left\{\xi^{(i)}\right\}, \xi^{(i)}=\left\{\xi^{(i)}(n)\right\}_{n=1}^{\infty}, \quad E_{\theta} \xi^{(i)}(n) \equiv 0, \\
i \in J \triangleq\{1,2, \ldots, k+1\} .
\end{gathered}
$$

Assume $x^{N}(n)=\phi_{\theta}(n / N)+\xi^{(i)}(n)$ when $\left[\theta_{i-1} N\right]<n \leq$ $\left[\theta_{i} N\right], i \in J$. The a posteriori change point problem is an estimate of change point time $\tau_{i}=\left[\theta_{i} N\right], i=1,2, \ldots, k$ of random sequence $X^{N}$. Therefore, the change point test problem translates into the consistent estimation problem of parameter vector $\theta$ according to the observation of $X^{N}$. For example, as to the change point problem with a mutation change point, when $k=1, \theta=\theta_{1}, \phi_{\theta}(t)=a I\left(t>\theta_{1}\right), a \neq 0$, where $I(\cdot)$ is an indicator function, the following statistics should be considered:

$$
\begin{array}{r}
Y_{N}(n)=\frac{1}{n} \sum_{k=1}^{n} x^{N}(k)-\frac{1}{N-n} \sum_{k=n+1}^{N} x^{N}(k), \\
n=1,2, \ldots, N-1 .
\end{array}
$$

In the interval $[0,1]$, use a sequence of points $((n / N)$, $\left.Y_{N}(n)\right)$ obtained to construct a continuous random process $y_{N}(t)$ by liner interpolation, the random point in the set $M_{N}$ is the estimate $\hat{\theta}_{N}$ of parameter $\theta$, where

$$
M_{N}=\left\{\tilde{t} \in[\alpha, \beta]:\left|y_{N}(\tilde{t})\right|=\max _{\alpha \leq t \leq \beta}\left|y_{N}(t)\right|\right\} .
$$

Under certain conditions, for random $\varepsilon>0, P\left\{\left|\widehat{\theta}_{N}-\theta\right|>\right.$ $\varepsilon\}=O\left(H_{N}\right)$ exists. Meanwhile, when certain conditions are satisfied, $\widehat{\theta}_{N} \stackrel{\text { a.s. }}{\longrightarrow} \theta$. Assuming $\left\{\xi^{(1)}(n)\right\}$ and $\left\{\xi^{(2)}(n)\right\}$ are two strict and stationary independence random sequences, when certain conditions are satisfied, sequence $\sigma_{i}^{2}=E_{\theta}\left(\left\{\xi^{(i)}(1)\right\}\right)^{2}+$ $2 \sum_{k=2}^{\infty} E_{\theta} \xi^{(i)}(1) \xi^{(i)}(k), i=1,2$ is of absolute convergence. And if $\sigma_{i}^{2}>0, i=1,2$, on the continuity interval $C[\alpha, \beta]$ :

$$
\sqrt{N}\left(y_{N}(t)-E_{\theta} y_{N}(t)\right) \stackrel{d}{\longrightarrow} z(t),
$$

where

$$
z(t)= \begin{cases}{[t(1-t)]^{-1}\left[\sigma_{1} W_{1}(t)-t\left(\sigma_{1} W_{1}(\theta)+\sigma_{2}\left(W_{2}(1)-W_{2}(\theta)\right)\right)\right]} & t \leq \theta \\
{[t(1-t)]^{-1}\left[\begin{array}{c}
\sigma_{1} W_{1}(\theta)+\sigma_{2}\left(W_{2}(t)-W_{2}(\theta)\right) \\
-t\left(\sigma_{1} W_{1}(\theta)+\sigma_{2}\left(W_{2}(1)-W_{2}(\theta)\right)\right)
\end{array}\right]} & t \geq \theta\end{cases}
$$

where $W_{1}$ and $W_{2}$ are two standard and independent Wiener processes.

When $\varepsilon^{(1)} \equiv \varepsilon^{(2)}, W_{1} \equiv W_{2} \triangleq W$, and $\sigma_{1} \equiv \sigma_{2} \triangleq \sigma, z(t)$ can be simplified as

$$
z(t)=\sigma[t(1-t)]^{-1}[W(t)-t W(1)] .
$$

The above method is constructed in consideration of the basic type of change point. Meanwhile, the change point test method of basic change point problem mentioned above can be spread to the change point test problem of any probability characteristics.

3.3. Asymptotic Analysis of Basic Change Point Test Method. Through the asymptotic analysis of first and second types of probability errors and estimated probability error, it is able to choose the most optimal adjustment parameter in the family to meet the requirement of change point test quality in practice. Assuming $X=\{x(n)\}_{n=1}^{N}$ is an observation of a random sequence, the following form of statistics family should be considered:

$$
Y_{N}(n, \delta)=\left[\frac{n}{N}\left(1-\frac{n}{N}\right)\right]^{\delta}\left[\frac{1}{n} \sum_{k=1}^{n} x(k)-\frac{1}{N-n} \sum_{k=n+1}^{n} x(k)\right]
$$

where $0 \leq \delta \leq 1$ and $n=1,2, \ldots, N-1$.

When $\delta=0, Y_{N}(n)$ is the statistics. When $\delta=1 / 2$, $Y_{N}(n)$ is the maximum likelihood statistics constructed by Hinkly [12] for Gaussian sequence. When $\delta=1, Y_{N}(n)$ is the nonparametric statistics adopted by Deshayes and Picard [13, 14]. Assume the observed sequence $X$ is from the following generating scheme:

$$
x(t)=r+h I(t>\theta T)+\sigma \dot{W}(t), \quad 0 \leq t \leq T,
$$


where $0<a \leq \theta \leq b<1, \sigma>0, r \in \mathbb{R}^{1}, h \in \mathbb{R}^{1}$, and $h \neq 0, \dot{W}(t)$ is Gaussian white noise, and $W(t)$ is the standard Wiener process. For continuous time sequence, the statistics $Y_{N}(n)$ can be expressed as

$$
Y_{T}(t)=\left[\frac{t}{T}\left(1-\frac{t}{T}\right)\right]^{\delta} Y_{T^{\prime}}(t),
$$

where $Y_{T^{\prime}}(t)=(1 / t) \int_{0}^{t} x(s) d s-(1 /(T-t)) \int_{t}^{T} x(s) d s$. In the interval of $[0,1], y_{T}(s)=Y_{T}(s T)$, so the maximum value point of process $\left|y_{T}(s)\right|$ on the interval of $[a, b]$ is the estimate $\widehat{\theta}_{T}$ of change point $\theta$.

The first type of error probability can be expressed as

$$
\alpha(T)=P\left\{\max _{a \leq s \leq b}\left|y_{T}(s)\right|>c \mid \theta=0\right\} .
$$

The second type of error probability can be expressed as

$$
\beta(T)=P\left\{\max _{a \leq s \leq b}\left|y_{T}(s)\right| \leq c \mid a \leq \theta \leq b\right\} .
$$

And estimated error probability can be expressed as

$$
\gamma(\varepsilon, T)=P\left\{\left|\widehat{\theta}_{T}-\theta\right|>\varepsilon \mid a \leq \theta \leq b\right\} .
$$

For the first type of error probability, the following equation exists:

$$
\lim _{T \rightarrow \infty} \frac{|\ln \alpha(T)|}{T}= \begin{cases}\frac{1}{2}\left(\frac{c}{\sigma}\right)^{2} \Delta^{2}, & 0 \leq \delta \leq \frac{1}{2}, \\ \frac{1}{2}\left(\frac{c}{\sigma}\right)^{2} 2^{4 \delta-2}, & \frac{1}{2}<\delta \leq 1,\end{cases}
$$

where $\Delta=\Delta(a, b)=\min \left([a(1-a)]^{0.5-\delta},[b(1-b)]^{0.5-\delta}\right)$. It is obvious that function $r(\delta) \triangleq \lim _{T \rightarrow \infty}(|\ln \alpha(T)| / T)$ is of monotone increasing on the interval of $0 \leq \delta \leq$ 1. So the asymptotic optimal method to reduce the first type of error probability is to assume $\delta=1$ and then $\lim _{T \rightarrow \infty}(|\ln \alpha(T)| / T)=2(c / \sigma)^{2}$.

For the second type of error probability, under certain conditions, the following formula exists:

$$
\lim _{T \rightarrow \infty} P\left\{\sqrt{T} \max _{a \leq s \leq b}\left(\left|y_{T}(s)\right|-|h| p^{\delta}\right)\right\}=\Phi\left(\frac{|h|\left(\lambda-p^{\delta}\right)}{\sigma p^{\delta-1 / 2}}\right) .
$$

It can be seen that the second type of error probability satisfies

$$
\beta(T) \sim \exp \left(-\frac{T h^{2}}{2 \sigma^{2}} \frac{\left(p^{\delta}-\lambda\right)^{2}}{p^{2 \delta-1}}\right) .
$$

Therefore, the asymptotic optimal method to reduce the second type of error probability is to assume $\delta=0$, so $\beta(T) \sim$ $\exp \left(-\left(T h^{2} / 2 \sigma^{2}\right) p(\theta)(1-\lambda)^{2}\right)$.
For estimated error probability, any $z>0$ exists:

$$
\begin{aligned}
\lim _{T \rightarrow \infty} P & \left\{T\left|\widehat{\theta}_{T}-\theta\right|>z\right\} \\
= & 1-(1-\mu(\delta, \theta)) G\left(\frac{|h| \rho_{1}(\delta, \theta)}{\sigma} p^{1-\delta}, z\right) \\
& -\mu(\delta, \theta) G\left(\frac{|h| \rho_{2}(\delta, \theta)}{\sigma} p^{1-\delta}, z\right),
\end{aligned}
$$

where $\mu(\delta, \theta) \triangleq \delta \theta+(1-\delta)(1-\theta), G(b, t)=2 \Phi(b \sqrt{t})-1-$ $2 b^{2} t \Phi(-b \sqrt{t})+2 b \sqrt{t / 2 \pi} \exp \left(-b^{2} t / 2\right), \Phi$ is a standard normal distribution function, $\rho_{1}(\delta, \theta)=[p(\theta)]^{\delta-1}[\delta \theta+(1-\delta)(1-\theta)]$, and $\rho_{2}(\delta, \theta)=p^{\delta-1}-\rho_{1}(\delta, \theta)$. When $T \rightarrow \infty$,

$$
\begin{aligned}
\gamma(\varepsilon, T) \sim & 2 \varepsilon T \frac{h^{2} p^{2-2 \delta}}{\sigma^{2}}(1-\mu(\delta, \theta)) \rho_{1}^{2}(\delta, \theta) \\
& \times \exp \left(-\frac{h^{2} \rho_{1}^{2}(\delta, \theta)}{2 \sigma^{2}} p^{2-2 \delta} \varepsilon T\right) \\
& +\mu(\delta, \theta) \rho_{2}^{2}(\delta, \theta) \exp \left(-\frac{h^{2} \rho_{2}^{2}(\delta, \theta)}{2 \sigma^{2}} p^{2-2 \delta} \varepsilon T\right) .
\end{aligned}
$$

Namely, when $T \rightarrow \infty$,

$$
\begin{aligned}
\frac{\ln |\gamma(\varepsilon, T)|}{T} \sim & \frac{h^{2} p^{2-2 \delta}}{2 \sigma^{2}} \varepsilon \min \left(\rho_{1}^{2}(\delta, \theta), \rho_{2}^{2}(\delta, \theta)\right) \\
= & \frac{h^{2} \varepsilon}{2 \sigma^{2}} \min \left\{[\delta \theta+(1-\delta)(1-\theta)]^{2},\right. \\
& {\left.[\delta(1-\theta)+\theta(1-\delta)]^{2}\right\} \triangleq R(\delta, \theta), }
\end{aligned}
$$

where $R(\delta, \theta)$ satisfies $\max _{0 \leq \delta \leq 1} \min _{a \leq \theta \leq b} R(\delta, \theta)=h^{2} \varepsilon / 8 \sigma^{2}$; namely, when $\delta=1 / 2$, the estimated error probability is the minimum. Therefore, selection appropriate parameter $\delta$ can improve the quality of change point test.

The nonparametric diagnosis method of crack behavior abnormality of concrete dam is constructed theoretically by the reduction of any change point problems, construction of basic nonparametric change point model, and asymptotic analysis of basic change point test method. And its basic ideas are first, to construct corresponding diagnosis sequence based on the initial time sequence of CMOD according to the reduction guidelines of change point problem; then, to construct statistics sequence according to the formula (11); finally, to diagnose whether the change point exists or not according to the formula (13) under the given level of significance test.

However, in practice, for the given CMOD sequence of concrete dam, it is hard to ensure that there is only one abnormality point upon crack abnormality, so the number of crack abnormality point is unknown. Therefore, the multiple change point diagnosis method of crack behavior abnormality of concrete dam is built combing with the asymptotic property of the statistics family below. 
3.4. Multiple Change Point Diagnosis Method of Crack Behavior Abnormality. For many existing change point test methods, if assuming the diagnosis sequence can be described by a mathematical model of limited parameter set, the methods belong to the scope of parametric change point test method [15-17]. When the phenomenological model of study object is known, the parametric method is effective. However, when the phenomenological model has model error during setting, the result obtained by parametric change point test method is doubtful. Therefore, this paper constructs corresponding nonparametric change point test method to diagnose the crack behavior abnormality of concrete dam which has less demand of prior information of diagnosis sequence; that is to say, the nonparametric method does not require the prior information of probability distribution of diagnosis sequence [18]. Furthermore, for the given level of significance test, this method can almost test the abnormality characteristics of random crack behavior. Assuming a random sequence observation $X^{N}=\left\{x^{N}(n)\right\}_{n=1}^{N}, N=1,2, \ldots$ satisfies

$$
\begin{array}{r}
x^{N}(n)=\phi\left(\frac{n}{N}\right)+\varepsilon(n), \quad\left[\theta_{i-1} N\right]+1 \leq n \leq\left[\theta_{i} N\right], \\
(i=1,2, \ldots, k+1),
\end{array}
$$

where $\phi(t)=\sum_{i=1}^{k} a_{i} I\left(\theta_{i-1} \leq t \leq \theta_{i}\right)+a_{k+1} I\left(\theta_{k} \leq t \leq 1\right)$ and $a_{i} \neq a_{i-1}, i=1,2, \ldots, k$.

Assume $\min _{1 \leq i \leq k+1}\left(\theta_{i}-\theta_{i-1}\right) \geq \delta$ and $\min _{1 \leq i \leq k}\left(a_{i+1}-a_{i}\right) \geq$ $h$, where $0<\delta<1 / 2$ and $h>0$. And assuming $\{\varepsilon(n)\}$ is independent identically distributed random variables sequence, $E_{\theta} \varepsilon(n)=0$ and $D \varepsilon(n)=\sigma^{2}$ satisfies. The model (28) is the corresponding basic nonparametric change point model in case of multiple change points existed. For the actual CMOD in concrete dam, it can be turned into the form of this model by constructing appropriate diagnosis sequence. Therefore, this paper analyzes the multiple change point of this model and builds corresponding multiple change point diagnosis method of crack behavior abnormality of concrete dam.

The asymptotic analysis of basic change point test method shows that when $\delta=1$, the first type of error probability is minimum; namely, the false alarm probability is minimum; when $\delta=0$, the second type of error probability is minimum; namely, the false dismissal probability is minimum; when $\delta=1 / 2$, the estimated error probability of change point is minimum; namely, the probability of change point estimated value far away from the actual change point position is minimum. Therefore, selecting different $\delta$ value in different change point test phase can improve the precision and credibility of the estimated result of change point [19]. The basic steps of multiple abnormality point diagnosis method of crack behavior abnormality of concrete dam are as follows.

(1) Construction of the diagnosis sequence from the original CMOD sequence: construct diagnosis sequence from original signal to make the change of various characteristics of original signal convert into the change of diagnosis sequence mathematical expectation.
(2) Homogeneity test of diagnosis sequence: calculate the statistics $Y_{N}(n, 1), 1 \leq n \leq N-1$ from diagnosis sequence. If

$$
\max _{1 \leq n \leq N-1}\left|Y_{N}(n, 1)\right| \leq C
$$

is true, this diagnosis sequence should be deemed as homogeneity; that is to say, there is no abnormality of crack behavior. If it is not workable, this diagnosis sequence should be deemed as nonhomogeneity; that is to say, this sequence is of crack behavior abnormality point. Go to the next step.

In the formula (29), $C$ is the threshold value of crack behavior abnormality point test which relies on the specified false alarm probability, sample size, sample variance, and correlation coefficient of sample where the false alarm probability is specified in advance, the smaller this parameter value, the more abnormality point will be of missing test. In this phase, to not miss testing any possible abnormality point, the false alarm probability should be bigger.

(3) Initial estimation of crack behavior abnormality point: the point $n_{1}$ that makes the statistics $\left|Y_{N}(n, 1)\right|$ global maximum can be used as the estimation of first abnormality point. This point divides the diagnosis sequence into two subsample set including $Z_{1}$ : $1 \leq$ $n \leq n_{1}-[\varepsilon N]$ and $Z_{2}: n_{1}+[\varepsilon N] \leq n \leq N$ where $\varepsilon$ is a smaller value relying on the sample size and the maximum value in statistics. Then, test the homogeneity of each subsample $Z_{1}$ and $Z_{2}$ by the method in step (2) and estimate the abnormality point by the method in step (3). Repeat above steps until each sample in the subsample set is homogeneous. A sequence of initial estimation $n_{1}, n_{2}, \ldots, n_{k}$ of abnormality points is obtained by steps (2) and (3) where $k$ is the initial estimation number of abnormality points.

(4) Eliminating suspicious crack behavior abnormality point: redivide the diagnosis sequence to make each subsample set only contain an abnormality point $n_{i}$ of initial estimation, that is to say

$$
\begin{gathered}
X_{1}: 1 \leq n \leq n_{1}+0.5\left(n_{2}-n_{1}\right), \\
X_{k}: n_{k-1}+0.5\left(n_{k}-n_{k-1}\right) \leq n \leq N, \\
X_{s}: n_{s-1}+0.5\left(n_{s}-n_{s-1}\right) \leq n \leq n_{s} \\
\quad+0.5\left(n_{s+1}-n_{s}\right), \quad s=2, \ldots, k-1 .
\end{gathered}
$$

Conduct homogeneity test of each subsample set according to step (2). However, the false alarm probability should be smaller. If a certain subsample set is tested to be homogeneous, the corresponding abnormality points in the subsample set should be eliminated.

(5) Final estimation of crack behavior abnormality point: after step (4), for the remaining subsample set $X_{i}$ with a size of $N_{i}$, calculate the statistics $Y_{N}(n, 1 / 2)$, and the corresponding point of maximum value of the 
statistics is the final estimation of the $i$ abnormality point. In the above abnormality point test method, the determination of threshold $C$ is the key. To calculate the threshold $C$, the homogeneity assumption of sequence should be workable. Then, according to the functional limit theorem of random sequence [20], the following formula exists under certain conditions

$$
\lim _{N \rightarrow \infty} \sqrt{N} Y_{N}([N t], 1) \longrightarrow \sigma W^{o}(t), \quad t \in[0,1]
$$

where $\sigma$ makes the sequence $\{\varepsilon(k)\}$ meet $\lim _{n \rightarrow \infty}(1 /$ $n) E\left(\sum_{k=1}^{n} \varepsilon(k)\right)^{2}=\sigma^{2}$ and $W^{o}(\cdot)$ is standard Brownian bridge:

$$
\begin{aligned}
& \lim _{N \rightarrow \infty} P\left\{\sqrt{N} \max _{1 \leq n \leq N-1}\left|Y_{N}(n, 1)\right|>C\right\} \\
& =P\left\{\max _{0 \leq t \leq 1}\left|W^{o}(t)\right|>\frac{C}{\sigma}\right\} \\
& =2 \sum_{k=1}^{\infty}(-1)^{k+1} \exp \left[-2 k^{2}\left(\frac{C}{\sigma}\right)^{2}\right] \equiv F(C) .
\end{aligned}
$$

When false alarm probability $P_{\text {f.a. }}$ is given, threshold $C$ can be calculated by the following formula:

$$
F(C)=P_{\text {f.a. }} \text {. }
$$

It can be seen from the above analysis that the threshold $C$ relies on $\sigma$ and $\sigma$ is unknown. To solve this problem, Piryatinska et al. [21] adopts the following method to estimate this threshold. First, when the false alarm probability $P_{\text {f.a. }}$ is given, assuming $\sigma=1$, the initial estimation of threshold $C^{\prime}$ can be obtained according to the formulae (34) and (35). Then, divide the diagnosis sequence into two subsample sets including $Z_{1}: 1 \leq n \leq n_{1}-[\varepsilon N]$ and $Z_{2}: n_{1}+[\varepsilon N] \leq$ $n \leq N$ by steps (2) and (3), calculate the mean value of each subsample set, and deduct the corresponding mean value from the subsample set to form a new sample set by merging. Finally, calculate the empiric variance of the sample set as the estimation $\widehat{\sigma}^{2}$ of $\sigma^{2}$. So the estimation of threshold $C$ is

$$
\widehat{C}=\widehat{\sigma} N^{-1 / 2} C^{\prime} \text {. }
$$

It can be seen from the basic principle of the method that when there is no or only one abnormality point in the diagnosis sequence, the estimation $\widehat{\sigma}^{2}$ is more precise and the estimation $\widehat{C}$ is much reliable. However, when there are multiple abnormality points in the diagnosis sequence, the error of estimation $\widehat{\sigma}^{2}$ obtained by this method is larger and the estimation $\widehat{C}$ is suspicious. Meanwhile, it is assumed initially that $\sigma=1$ can affect the threshold estimation $\widehat{C}$ to a certain degree.

In consideration of the problems existed, this paper improves this method and its basic ideas are under an assumed $\sigma$, estimate the abnormality points in the diagnosis sequence, then use empiric variance estimation $\sigma=\sigma_{e}$. according to similar method, and obtain corresponding threshold estimation $\widetilde{C}$ according to the formula (36). Then

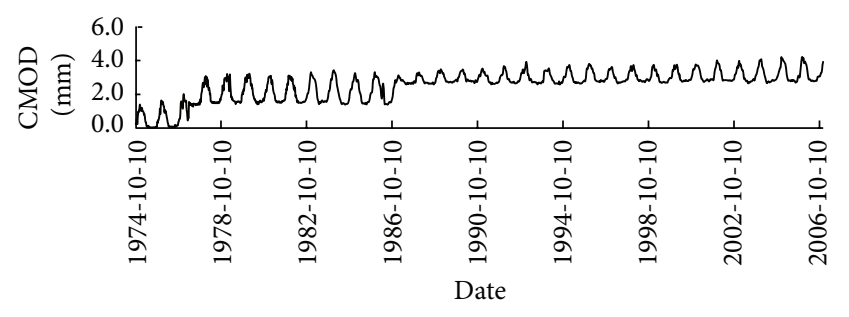

FIgURE 1: Time sequence curve of the measured CMOD.

analyze the abnormality points of diagnosis sequence again by this threshold, in this cycle, until the abnormality point estimation for two times is the same, $\sigma$ estimation satisfies $\widehat{\sigma}=\sigma_{e}$, and threshold estimation is $\widehat{C}=\widetilde{C}$. This improvement can eliminate the influence of multiple abnormality points and assumed $\sigma$ on threshold to a great extent.

\section{Project Case}

Conduct abnormality diagnosis of the crack near $105 \mathrm{~m}$ elevation at the downstream face of a gravity arch dam based on the nonparametric change point diagnosis method of crack behavior abnormality of concrete dam. This dam is a concrete gravity arch dam of concentric variable radius with a crest elevation of $126.3 \mathrm{~m}$ and maximum dam height of $76.3 \mathrm{~m}$ and has 28 dam sections from left to right. It takes 12 years from dam pouring to substantial completion and is constructed by three phases. During Phase II concrete pouring, as the level rising speed is fast and interval of concrete lift is short, the contraction distortion of Phase II concrete is strongly constrained by Phase I concrete to make the top of Phase I concrete have cracks from $5^{\#}$ dam block to $28^{\#}$ dam block with a length of more than $300 \mathrm{~m}$. So, the dam blocks of $14^{\#} \sim 20^{\#}$ were of cross-seam joint bar grouting in 1973 and of epoxy resin grouting in 1987. This paper analyzes the 1680 CMOD monitoring data of joint meter inbuilt in $18^{\#}$ dam block from October 10, 1974 to December 18, 2006 (as shown in Figure 1), so as to diagnose the abnormality conditions of crack behavior.

Upon the analysis of given CMOD sequence of concrete dam by adopting nonparametric change point diagnosis criteria, construct diagnosis sequence according to the original sequence first. And the CMOD means and amplitudes are the two essential characteristics where the mean value presents the base point of change of CMOD, namely, the average level of CMOD, and the amplitudes represent the amplitude of variation of crack under load in one cycle. Therefore, when there is change point in CMOD means or amplitudes, the crack of corresponding moment is deemed as abnormality. Based on this, this paper analyzes the crack behavior abnormality according to the diagnosis sequence of CMOD means, amplitudes, and correlation functions.

The diagnosis sequence based on CMOD means can be expressed as

$$
x_{i}=\frac{1}{\tau} \sum_{t=i}^{i+\tau} \mathrm{CMOD}_{t}, \quad i=1,2, \ldots, n-\tau .
$$




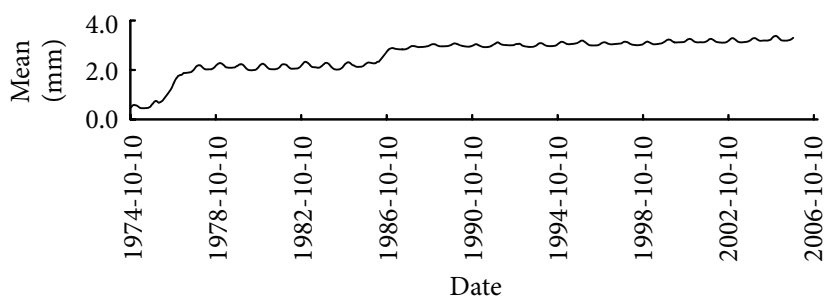

FIgURE 2: Diagnostic curve of the CMOD means.

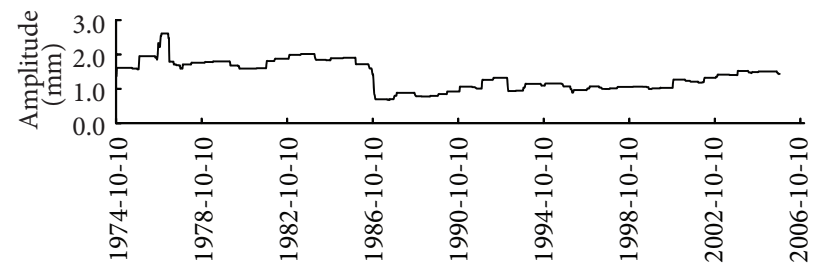

Figure 3: Diagnostic curve of the CMOD amplitudes.

The diagnosis sequence based on CMOD amplitudes can be expressed as

$$
x_{i}=\max _{i \leq t_{1}<t_{2} \leq i+\tau}\left|\mathrm{CMOD}_{t_{1}}-\mathrm{CMOD}_{t_{2}}\right|, \quad i=1,2, \ldots, n-\tau,
$$

where $\tau$ is time delay.

The diagnosis sequence based on CMOD correlation functions can be expressed as

$$
\begin{gathered}
x_{i}=\mathrm{CMOD}_{i} \mathrm{CMOD}_{i+\tau}, \\
i=1,2, \ldots, n-\tau ; \quad \tau=0,1, \ldots, \tau_{m},
\end{gathered}
$$

where $\tau_{m}$ is time delay.

The CMOD sequence and the diagnosis sequence of its corresponding means, amplitudes, and correlation function in this case should refer to Figures 2, 3, and 4.

This paper analyzes the diagnosis sequence according to the nonparametric change point diagnosis method of crack behavior abnormality built where Figure 5 is the diagnosis process of crack behavior abnormality based on the diagnosis sequence of CMOD means which is similar with the abnormality diagnosis process of diagnosis sequence based on CMOD amplitudes and correlation functions and not repeated here. Tables 1,2 , and 3 are the abnormality diagnosis results of diagnosis sequence based on CMOD means, amplitudes, and correlation functions.

For the diagnosis sequence of CMOD means, it can be seen from Figure 2 that the CMOD means have two obvious change points during the period of January 15, 1976 to November 25, 1976 and September 26, 1985 to August 28,1986 , that is to say, the CMOD means have abnormality. Meanwhile, the CMOD means have increased since 1987; that is to say, the CMOD means increase gradually. However, it can be seen from Table 1 that upon the abnormality analysis of the diagnosis sequence of whole means, the abnormality point of CMOD means diagnosed first occurred on August

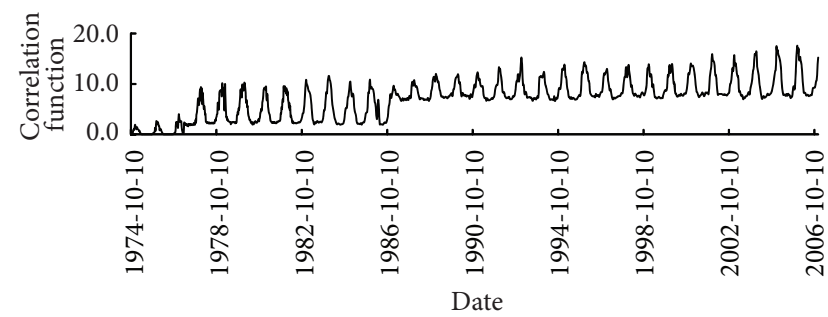

FIgURE 4: Diagnostic curve of the CMOD correlation functions.

28,1986 which divided the diagnosis sequence of means into two subsequences. Conduct abnormality diagnosis of each subsequence and the abnormality points of two subsequences occurred on May 5, 1977 and October 20, 1994. The final estimation time of the three abnormality points was on August 7, 1986, September 15, 1977 and October 6, 1994 which was similar to the observation of abnormality point in the diagnosis sequence of means. The abnormality diagnosis results of diagnosis sequence based on CMOD amplitudes and correlation functions are also similar.

As analyzed above, the nonparametric change point diagnosis method of crack abnormality built in this paper can diagnose the abnormality condition of crack behavior. The diagnosis result not only contains obvious abnormality point of crack behavior, but also is of good diagnosis effect on the abnormality point of crack behavior that is not easy to find.

\section{Conclusion}

(1) Whether the concrete dam crack expands or not is an important subject of safety performance evaluation of concrete dam, and the study on the abnormality diagnosis of crack behavior of concrete dam has become the hot spot and difficult problems in hydraulic structure safety. However, as the functional relationship between influencing factor and effect size of crack is set in advance and the random noise item of model should satisfy the normality assumption, the abnormality diagnosis result of crack behavior based on the safety monitoring parametric statistics model of concrete dam crack is not reasonable enough and comprehensive.

(2) The internal relationship between the crack behavior abnormality of concrete dam and statistics change point theory is deeply analyzed to make the abnormality diagnosis method of crack behavior of concrete dam based on change point theory have complete theoretical basis from the perspective of structural instability of parametric statistics model and change in the distribution law of sequence of nonparametric statistics model based on the representation of crack behavior abnormality of concrete dam in parametric statistics model and nonparametric statistics model.

(3) The test of parameters change of any distribution functions is reduced as the basic type and method of 
TABLE 1: Abnormality diagnosis results of diagnostic time sequence of the CMOD means.

\begin{tabular}{|c|c|c|c|c|c|}
\hline \multicolumn{6}{|c|}{ Sequence boundary } \\
\hline $\begin{array}{l}\text { Original } \\
\text { sequence }\end{array}$ & $\begin{array}{l}\text { The first estimation } \\
\text { of initial } \\
\text { abnormality points }\end{array}$ & $\begin{array}{l}\text { The second } \\
\text { estimation of initial } \\
\text { abnormality points }\end{array}$ & $\begin{array}{l}\text { The third } \\
\text { estimation of initial } \\
\text { abnormality points }\end{array}$ & $\begin{array}{l}\text { The forth } \\
\text { estimation of initial } \\
\text { abnormality points }\end{array}$ & $\begin{array}{l}\text { Final estimation of } \\
\text { abnormality points }\end{array}$ \\
\hline $1974-10-10$ & $1974-10-10$ & $1974-10-10$ & $1974-10-10$ & 1974-10-10 & $1974-10-10$ \\
\hline \multirow[t]{16}{*}{$2005-10-20$} & $1986-08-28$ & 1977-05-05 & $1976-06-03$ & 1975-10-09 & 1975-10-09 \\
\hline & $2005-10-20$ & $1986-08-28$ & 1977-05-05 & 1976-06-03 & 1976-09-02 \\
\hline & & $1994-10-20$ & $1982-09-23$ & - & - \\
\hline & & $2005-10-20$ & $1986-08-28$ & 1977-05-05 & 1977-09-15 \\
\hline & & & 1988-09-15 & 1978-09-07 & 1979-03-01 \\
\hline & & & 1994-10-20 & $1982-09-23$ & $1982-09-23$ \\
\hline & & & 1999-10-07 & $1985-09-26$ & 1984-02-09 \\
\hline & & & $2005-10-20$ & $1986-08-28$ & 1986-08-07 \\
\hline & & & & 1987-10-01 & $1987-10-29$ \\
\hline & & & & 1988-09-15 & - \\
\hline & & & & - & - \\
\hline & & & & $1994-10-20$ & 1994-10-06 \\
\hline & & & & - & - \\
\hline & & & & 1999-10-07 & 1999-09-16 \\
\hline & & & & 2003-10-09 & $2003-10-16$ \\
\hline & & & & $2005-10-20$ & $2005-10-20$ \\
\hline
\end{tabular}

TABLE 2: Abnormality diagnosis results of diagnostic time sequence of the CMOD amplitudes.

\begin{tabular}{|c|c|c|c|c|c|}
\hline \multicolumn{6}{|c|}{ Sequence boundary } \\
\hline $\begin{array}{l}\text { Original } \\
\text { sequence }\end{array}$ & $\begin{array}{l}\text { The first estimation } \\
\text { of initial } \\
\text { abnormality points }\end{array}$ & $\begin{array}{l}\text { The second } \\
\text { estimation of initial } \\
\text { abnormality points }\end{array}$ & $\begin{array}{l}\text { The third estimation } \\
\text { of initial } \\
\text { abnormality points }\end{array}$ & $\begin{array}{l}\text { The forth estimation } \\
\text { of initial } \\
\text { abnormality points }\end{array}$ & $\begin{array}{l}\text { Final estimation of } \\
\text { abnormality points }\end{array}$ \\
\hline $1974-10-10$ & $1974-10-10$ & $1974-10-10$ & $1974-10-10$ & $1974-10-10$ & $1974-10-10$ \\
\hline \multirow[t]{16}{*}{$2005-10-20$} & $1986-10-16$ & 1982-03-04 & $1977-06-16$ & 1975-11-06 & 1975-11-06 \\
\hline & $2005-10-20$ & $1986-10-16$ & 1982-03-04 & $1977-06-16$ & $1977-03-31$ \\
\hline & & 2000-10-19 & $1985-12-19$ & 1980-02-07 & 1980-02-07 \\
\hline & & $2005-10-20$ & $1986-10-16$ & 1982-03-04 & $1981-10-22$ \\
\hline & & & 1990-11-01 & $1984-01-26$ & $1984-01-26$ \\
\hline & & & $2000-10-19$ & $1985-12-19$ & $1985-12-19$ \\
\hline & & & $2002-11-21$ & - & - \\
\hline & & & $2005-10-20$ & $1986-10-16$ & $1986-10-23$ \\
\hline & & & & $1987-11-19$ & 1987-10-08 \\
\hline & & & & 1990-11-01 & 1990-11-01 \\
\hline & & & & 1995-09-07 & 1996-01-04 \\
\hline & & & & $2000-10-19$ & $2000-10-19$ \\
\hline & & & & 2002-04-04 & 2002-04-04 \\
\hline & & & & $2002-11-21$ & $2002-11-14$ \\
\hline & & & & 2003-10-30 & 2003-10-30 \\
\hline & & & & $2005-10-20$ & $2005-10-20$ \\
\hline
\end{tabular}




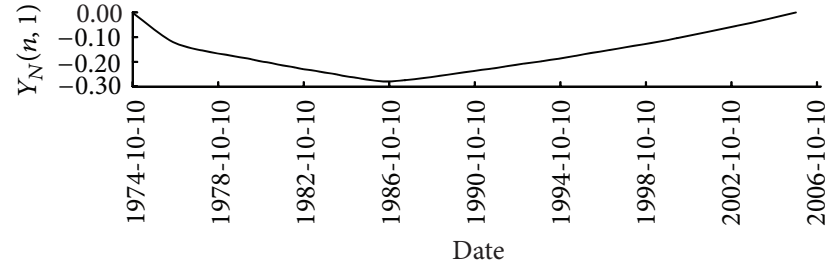

(a)

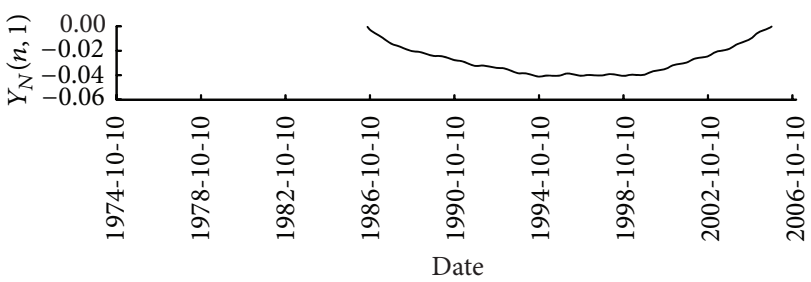

(c)

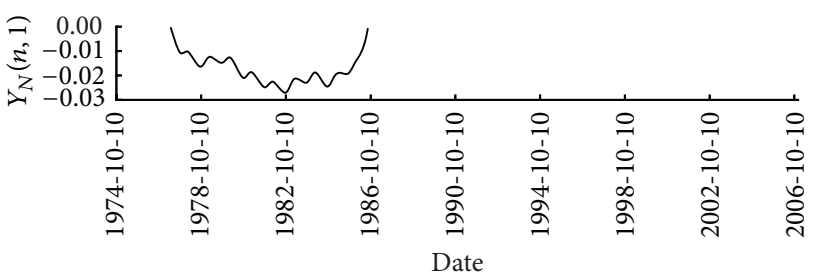

(e)

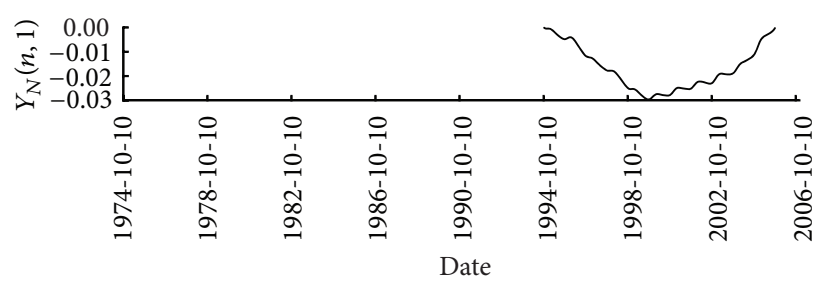

(g)

FiguRE 5: $Y_{N}(n, 1)$ curve of diagnostic time sequence of the CMOD means.

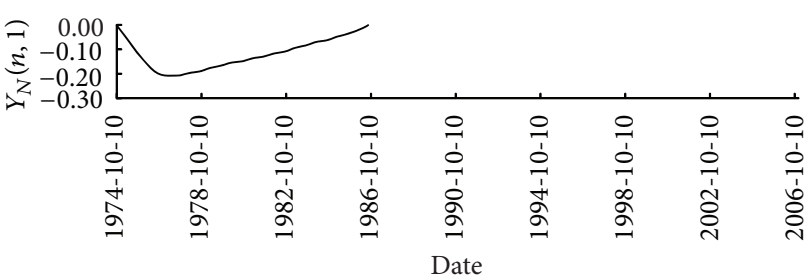

(b)

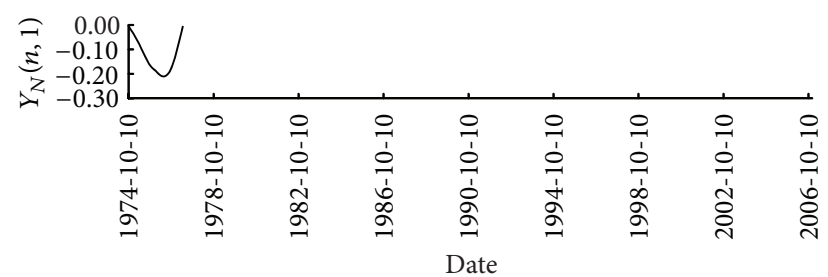

(d)

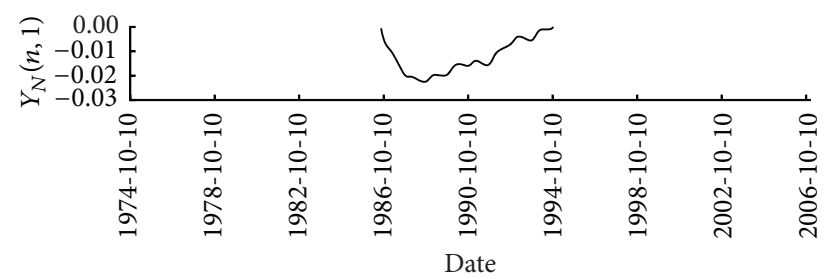

(f) change point test of sequence mathematical expectation by the reduction of change point problem and establishment of basic nonparametric change point model. The optimal adjustment parameter in the change point test statistics is selected by asymptotic analysis of basic change point test method to satisfy the requirement of change point test quality in practice. And the multiple change point diagnosis method of crack behavior abnormality of concrete dam is built considering that there may be multiple abnormality points in the crack behavior of concrete dam in practice. Finally, the nonparametric change point diagnosis method of crack behavior abnormality of concrete dam is built by integrating the above study results.

(4) The nonparametric diagnosis method of crack behavior abnormality of concrete dam can be applied in practical engineering. The diagnosis result contains obvious abnormality point of crack behavior and has good diagnosis effect of crack behavior abnormality point that is not easy to find, demonstrating the effectiveness and scientific rationality of built method. Meanwhile, the nonparametric change point diagnosis method of crack behavior abnormality of concrete dam is of complete theoretic basis, strong practicability, and wide application prospect in practical engineering.

(5) In accordance with the concept of dam safety monitoring, the research in this paper and many other researches on the abnormality diagnosis of crack behavior in concrete dam belong to the a posteriori category; that is, the crack monitoring data is regarded as given and the sample size is known and statistical central limit theory is used to diagnose crack behavior abnormality. However, in order to achieve the real-time monitoring and early warning of dam safety in practice, the concern about crack behavior abnormality of concrete dam is whether the current crack behavior is abnormal and whether the former monitoring model of crack safety is suitable 
TABLE 3: Abnormality diagnosis results of diagnostic time sequence of the CMOD correlation functions.

\begin{tabular}{|c|c|c|c|c|c|}
\hline \multicolumn{6}{|c|}{ Sequence boundary } \\
\hline $\begin{array}{l}\text { Original } \\
\text { sequence }\end{array}$ & $\begin{array}{l}\text { The first estimation } \\
\text { of initial } \\
\text { abnormality points }\end{array}$ & $\begin{array}{l}\text { The second } \\
\text { estimation of initial } \\
\text { abnormality points }\end{array}$ & $\begin{array}{c}\text { The third estimation } \\
\text { of initial } \\
\text { abnormality points }\end{array}$ & $\begin{array}{c}\text { The forth estimation } \\
\text { of initial } \\
\text { abnormality points }\end{array}$ & $\begin{array}{l}\text { Final estimation of } \\
\text { abnormality points }\end{array}$ \\
\hline $1974-10-10$ & $1974-10-10$ & $1974-10-10$ & $1974-10-10$ & $1974-10-10$ & $1974-10-10$ \\
\hline \multirow[t]{16}{*}{$2006-12-14$} & $1986-11-20$ & $1977-10-27$ & $1976-11-11$ & 1976-03-18 & $1975-11-20$ \\
\hline & $2006-12-14$ & $1986-11-20$ & $1977-10-27$ & $1976-11-11$ & $1976-11-11$ \\
\hline & & $1995-11-02$ & 1986-03-27 & - & - \\
\hline & & $2006-12-14$ & $1986-11-20$ & $1977-10-27$ & 1980-04-03 \\
\hline & & & $1988-10-27$ & $1982-11-11$ & $1983-11-10$ \\
\hline & & & 1995-11-02 & 1986-03-27 & $1985-11-07$ \\
\hline & & & $2000-10-26$ & - & - \\
\hline & & & 2006-12-14 & $1986-11-20$ & $1986-10-30$ \\
\hline & & & & 1988-03-24 & $1987-11-19$ \\
\hline & & & & $1988-10-27$ & $1988-10-20$ \\
\hline & & & & $1995-03-23$ & 1991-02-14 \\
\hline & & & & 1995-11-02 & 1995-11-09 \\
\hline & & & & 1996-03-28 & 1996-03-07 \\
\hline & & & & $2000-10-26$ & 1998-02-19 \\
\hline & & & & 2004-11-04 & 2001-11-01 \\
\hline & & & & $2006-12-14$ & $2006-12-14$ \\
\hline
\end{tabular}

for the current status, which corresponds to online diagnosis of crack behavior abnormality. The crack monitoring data is regarded as changing and the sample size is unknown in the online diagnosis. This will be the main research content of the authors and the research group in the future.

\section{Acknowledgments}

This work was supported by the Open Foundation of State Key Laboratory of Hydrology-Water Resources (Grant no. 2012490211), the doctoral scientific research fund of Northwest A\&F University in 2012 (Grant no. 2012BSJJ007), the Fundamental Research Funds for the Central Universities (Grant no. QN2013046), and the Key Project of the National Natural Science Foundation of China (Grant no. 51139001).

\section{References}

[1] D. H. Huang, Y. P. Song, and G. F. Zhao, "Study on crack characteristics of macrocrack concrete structure," China Civil Engineering Journal, vol. 33, no. 2, pp. 63-66, 2000.

[2] X. H. Li, H. Z. Xu, C. S. Gu et al., "Crack abnormality diagnosis based on wavelet analysis and cusp catastrophic model," Journal of Hohai University (Natural Science Edition), vol. 33, no. 3, pp. 301-305, 2005.

[3] T. F. Bao and H. Yu, "Detection of subcritical crack propagation for concrete dams," Science in China Series E, vol. 52, no. 12, pp. 3654-3660, 2009.

[4] C. S. Gu, Z. C. Li, and B. Xu, "Abnormality diagnosis of cracks in the concrete dam based on dynamical structure mutation,"
Science China Technological Sciences, vol. 54, no. 7, pp. 19301939, 2011.

[5] Z. C. Li, C. S. Gu, and Z. R. Wu, "Abnormality diagnosis of cracks in the concrete based on double crack tip opening displacement criterion," Science China Technological Sciences, vol. 56, no. 8, pp. 1915-1928, 2013.

[6] C. S. Gu and Z. R. Wu, Theory and Method for Security Monitoring and Control of Dam and Dam Foundation and Their Application, Hohai University Press, Nanjing, China, 2006.

[7] Z. R. Wu and C. S. Gu, Theory for Security Monitoring and Control of Hydraulic Structure and Its Application, Higher Education Press, Beijing, China, 2003.

[8] X. H. Li, Study on Evolvement Rule and Abnormality Diagnosis Method of Major Hydraulic Concrete Structure Crack, Hohai University, Nanjing, China, 2003.

[9] G. C. Chow, "Test of equality between sets of coefficients in two linear regressions," Econometrika, vol. 28, pp. 591-605, 1960.

[10] B. E. Brodsky and B. S. Darkhovsky, Nonparametric Methods in Change Point Problems, Kluwer Academic Publishers, Dordrecht, The Netherlands, 1993.

[11] A. N. Shiryaev, Probability, Nauka, Moscow, Russia, 1980.

[12] D. V. Hinkly, “Time-ordered classification," Boimetrika, vol. 59, pp. 509-523, 1972.

[13] J. Deshayes and D. Picard, "Off-line statistical analysis of change-point models using non parametric and likelihood methods," in Detection of Abrupt Changes in Signals and Dynamical Systems, vol. 77 of Lecture Notes in Control and Information Sciences, pp. 103-168, 1986.

[14] J. Deshayes and D. Picard, "Testing for a change-point in a statistical model," Tech. Rep., Universite de Paris-Sud, Orsay, France, 1981. 
[15] S. Shaban, "Change-point problem and two-phase regression: an annotated bibliography," International Statistical Review, vol. 48, pp. 83-93, 1980.

[16] P. Krishnaiah and B. Miao, "Review about estimation of change points," in Handbook of Statistics, P. Krishnaiah and C. R. Rao, Eds., vol. 7, pp. 375-402, Elsevier, Amsterdam, The Netherlands, 1988.

[17] P. Perron, "Dealing with structural breaks," Working Papers Series in Macroeconomics WP2005-017, Department of Economics, Boston University, 2005.

[18] M. Hollander and D. A. Wolfe, Nonparametric Statistical Methods, Wiley, New York, NY, USA, 1973.

[19] B. Brodsky, B. Darkhovsky, A. Y. Kaplan, and S. Shishkin, "A nonparametric method for the segmentation of the EEG," Computer Methods and Programs in Biomedicine, vol. 60, no. 2, pp. 93-106, 1999.

[20] M. Peligrad, "Invariance principles for mixing sequences of random variables," Annals of Probability, vol. 10, no. 4, pp. 968981, 1982.

[21] A. Piryatinska, G. Terdik, W. A. Woyczynski, K. A. Loparo, M. S. Scher, and A. Zlotnik, "Automated detection of neonate EEG sleep stages," Computer Methods and Programs in Biomedicine, vol. 95, no. 1, pp. 31-46, 2009. 


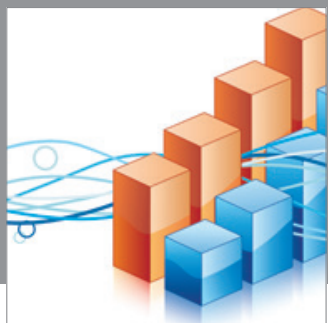

Advances in

Operations Research

mansans

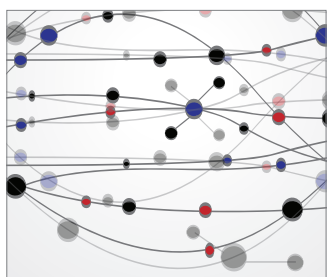

The Scientific World Journal
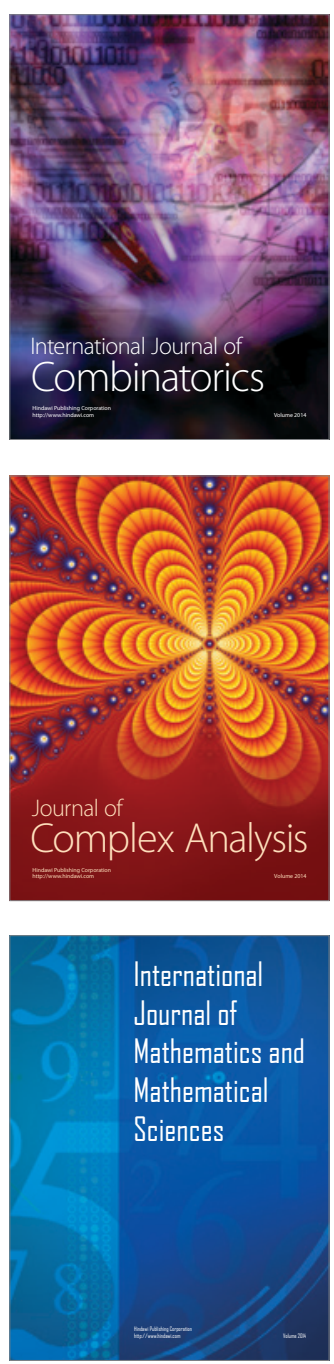
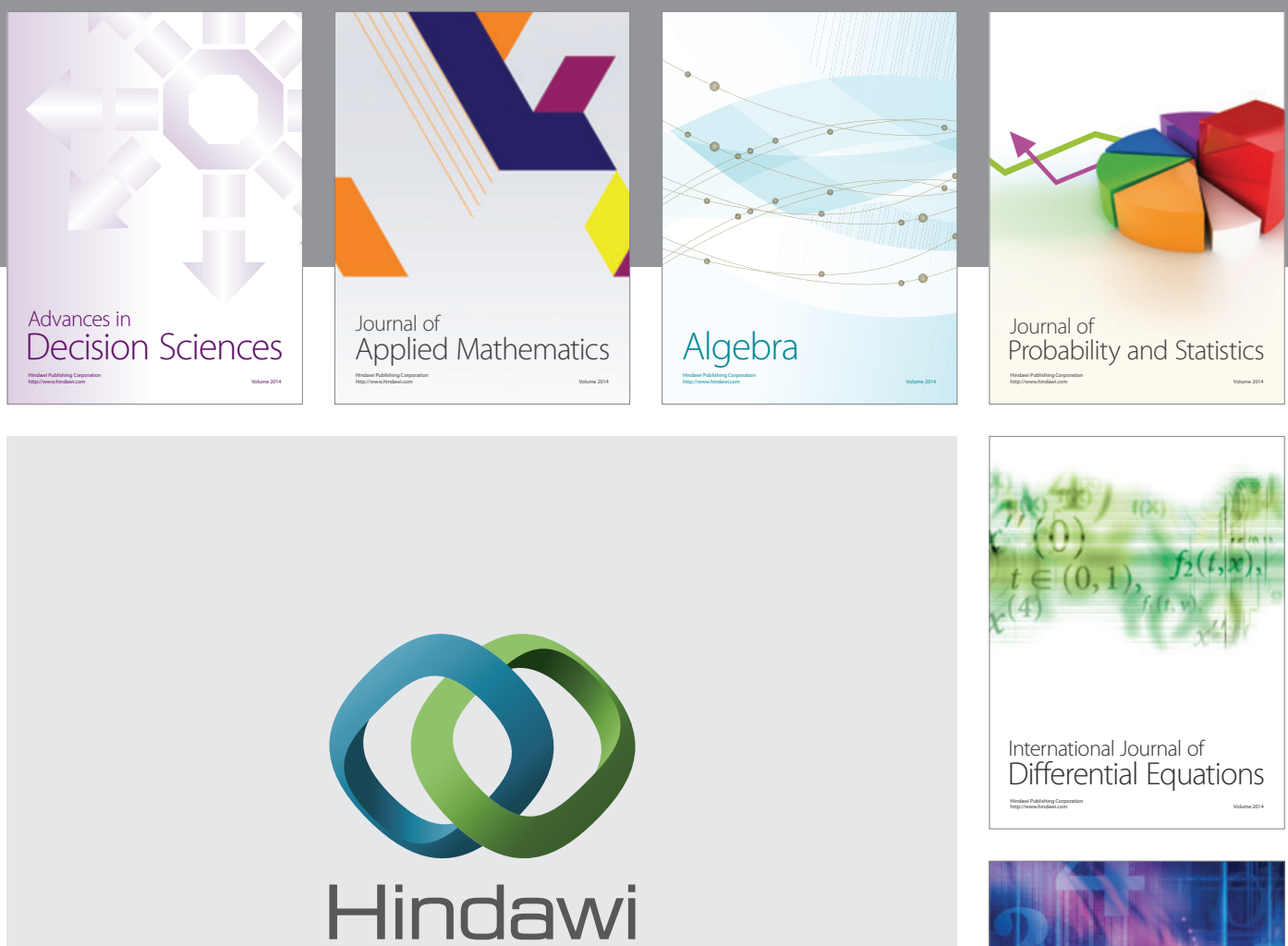

Submit your manuscripts at http://www.hindawi.com
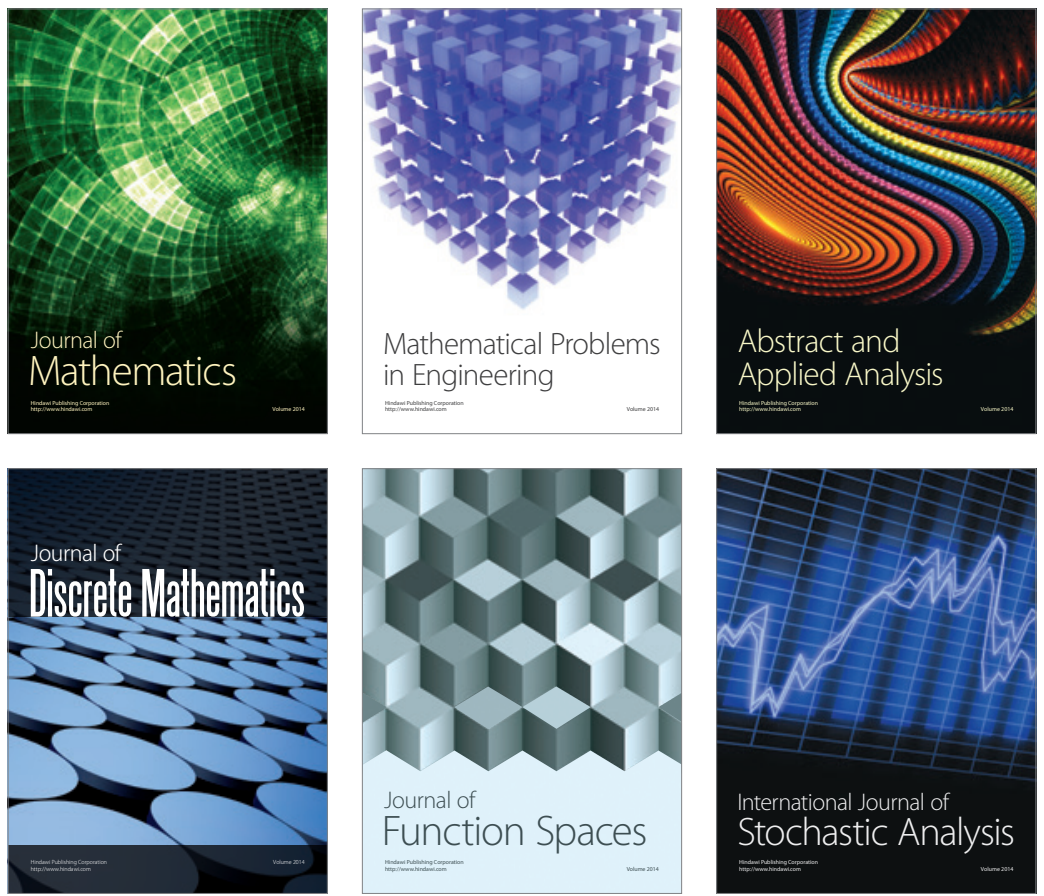

Journal of

Function Spaces

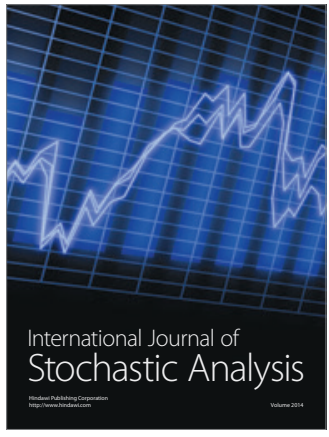

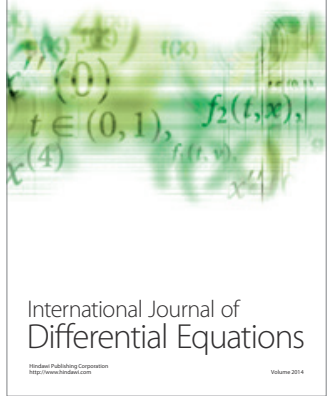
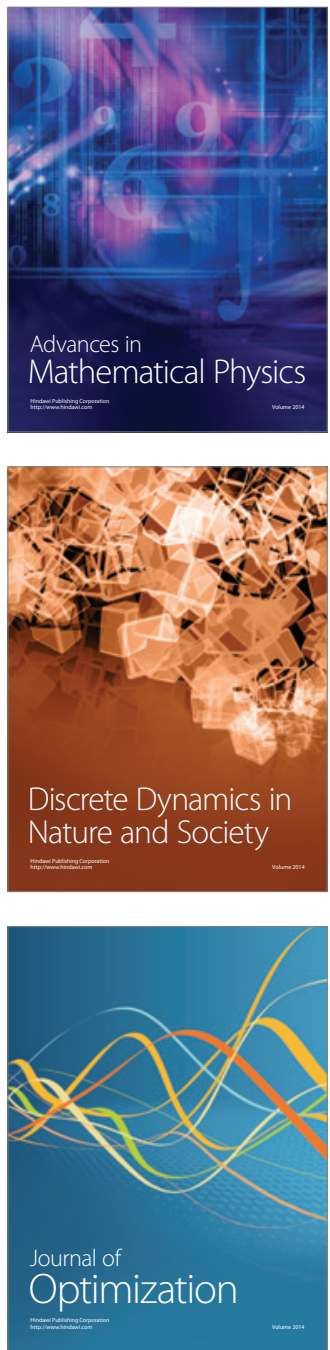